\title{
Correction to: High-temperature acclimation strategies within the thermally tolerant endosymbiont Symbiodinium trenchii and its coral host, Turbinaria reniformis, differ with changing $\mathrm{pCO}_{2}$ and nutrients
}

\author{
Kenneth D. Hoadley ${ }^{1}$. D. Tye Pettay ${ }^{1} \cdot$ Andréa G. Grottoli $^{2} \cdot$ Wei-Jun Cai ${ }^{1,3} \cdot$ Todd F. Melman $^{4} \cdot$ Stephen Levas ${ }^{2,8}$. \\ Verena Schoepf $^{2,7} \cdot$ Qian Ding ${ }^{3,9} \cdot$ Xiangchen Yuan $^{5}$ - Yongchen Wang ${ }^{3} \cdot$ Yohei Matsui $^{2}$ • Justin H. Baumann ${ }^{2,6}$. \\ Mark E. Warner ${ }^{1}$
}

Published online: 30 September 2020

(c) Springer-Verlag GmbH Germany, part of Springer Nature 2020

\section{Correction to: Mar Biol (2016) 163:134 https://doi.org/10.1007/s00227-016-2909-8}

In the original article, the light cycle was indicated as 12:12 $\mathrm{h}$ light:dark. However, this was in error and the actual cycle was 10:14 h light:dark. Additionally, there was a conversion error in calculating net production and LEDR as depicted in Figs. 2 and 3. This was strictly a conversion error and has no impact on the statistical analysis or interpretation of the data. The corrected panels from Fig. 2 and 3 are as follows.

The original article can be found online at https://doi.org/10.1007/ s00227-016-2909-8.

Kenneth D. Hoadley

khoadley@udel.edu

School of Marine Science and Policy, University of Delaware, Lewes, DE, USA

2 School of Earth Sciences, The Ohio State University, Columbus, OH, USA

3 Department of Marine Sciences, University of Georgia, Athens, GA, USA

4 Reef Systems Coral Farm, New Albany, OH, USA

5 Key Laboratory of Tropical Marine Bio-Resources and Ecology, Guangdong Provincial Key Laboratory of Applied Marine Biology, South China Sea Institute of Oceanology, Chinese Academy of Sciences, Guangzhou, China

6 Department of Marine Sciences, University of North Carolina, Chapel Hill, NC, USA

7 ARC Centre of Excellence for Coral Reef Studies, UWA Oceans Institute and School of Earth and Environment, University of Western Australia, Crawley, WA, Australia

8 Department of Geography and Environmental Science, Villanova University, Villanova, PA, USA

9 Ocean College, Zhejiang University, Hangzhou, China 

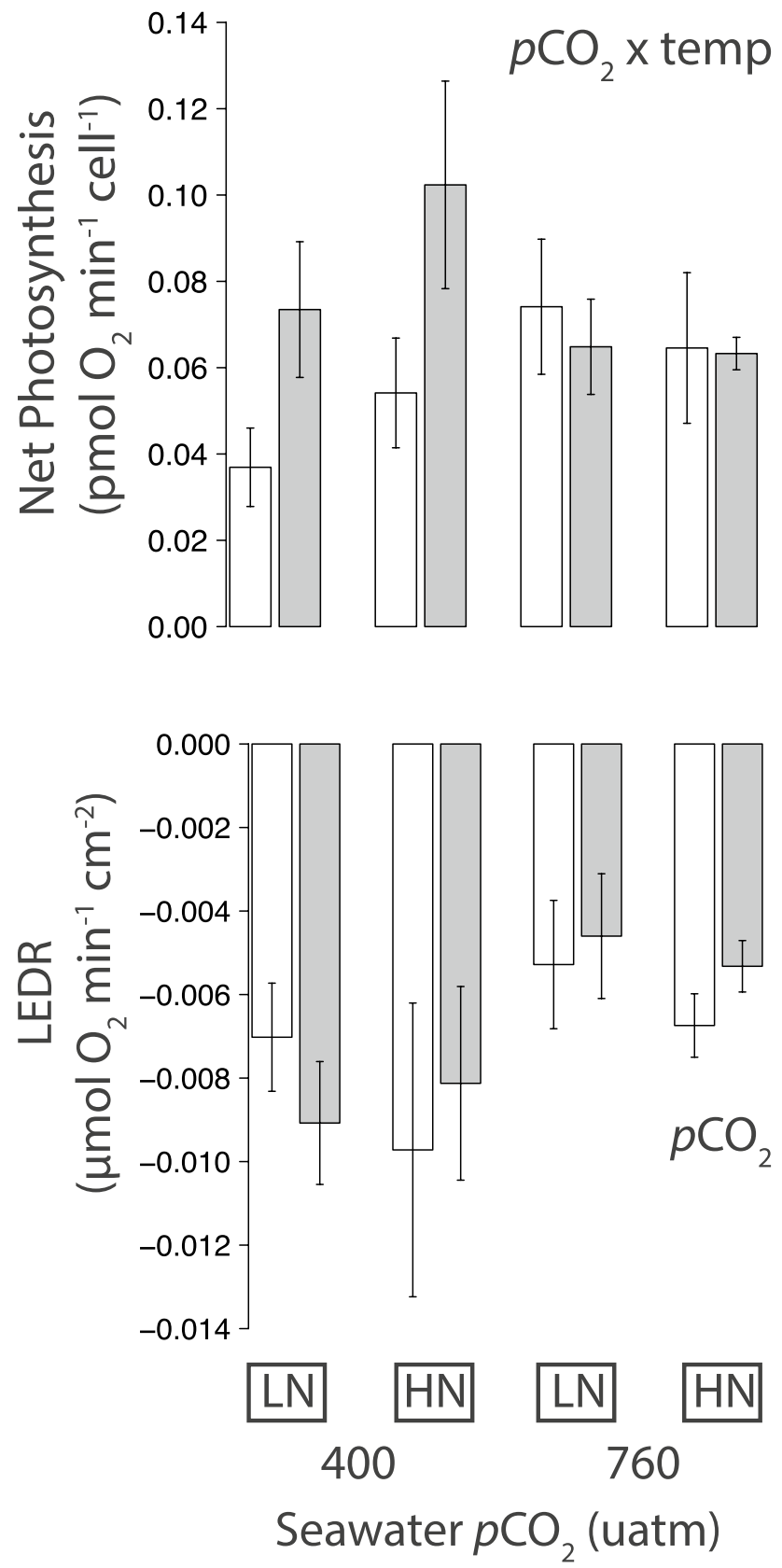

Average ( $\pm 1 \mathrm{SE})$ net photosynthesis cell ${ }^{-1}$ (top) and LEDR (bottom) at two $p \mathrm{CO}_{2}$ levels, nutrient concentrations ( $\mathrm{LN}=$ low nutrients, $\mathrm{HN}=$ high nutrients) and $26.5^{\circ} \mathrm{C}$ (light bars) or $31.5^{\circ} \mathrm{C}$ (dark bars). For each panel, the designations 'temp', ' $p \mathrm{CO}_{2}{ }^{\prime}$ and 'nutr' indicate significant temperature, $p \mathrm{CO}_{2}$, nutrient concentration, or their interactive effects (multifactorial ANOVA results in Table S1). $n=5-6$ per average.

Publisher's Note Springer Nature remains neutral with regard to jurisdictional claims in published maps and institutional affiliations. 